(A)

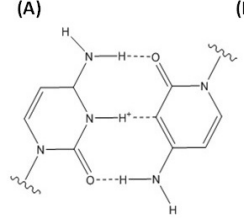

(C)

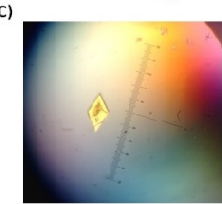

Figure 1. (A) Cytosine $e^{+}$cytosine base pairing in i-motifs. (B) Schematic diagrams of tetramolecular (left) bimolecular (middle) and intramolecular (right) $\mathrm{i}$-motifs. (C) $30 \times 25 \times 15$ micron crystal of $\mathrm{d}(\mathrm{CCCT})_{4}$

Keywords: i-motif, DNA, biophysical

\section{MS12-P5 Crystallography Platform at the Pasteur Institute}

Ahmed Haouz ${ }^{1}$, Patrick Weber ${ }^{1}$, Cedric Pissis ${ }^{1}$, Rafael Navaza ${ }^{1}$, Frederick Saul ${ }^{1}$

1. Institut Pasteur, PF crystallography, CNRS-UMR 3528, 25 Dr Roux, 75724, Paris France

email: ahaouz@pasteur.fr

The goal of the crystallography platform is to provide research teams working in the field of macromolecular crystallography at Institut Pasteur with the expertise and technology for high throughput crystallization, X-ray diffraction measurements, and crystallographic computing as a core facility. Our second mission is to offer expertise in bio-crystallography, from crystallization of selected targets to resolution of 3D crystal structures by participating as a partner in research projects involving structural studies of single proteins and protein complexes. These projects arise from direct collaboration with research groups at Institut Pasteur and outside organisations. Depending on the expertise of the users, three options can be offered: service provision, instrument allocation, and scientific collaboration. Service provision, which corresponds to the automated crystallization experiments performed in standard conditions, is the option used by the crystallographers. If initial crystallization trials are successful, the platform assists users to reproduce and optimize the crystallization conditions in order to obtain suitable crystals for X-ray data collection. Since 2010, the platform has been involved in more than 24 scientific collaborations in association with 14 research units from 8 scientific departments of the Institut Pasteur and 6 laboratories from other institutions (French or foreign), leading to our co-authorship of 32 peer-reviewed publications. These projects cover many disciplines related to infectious diseases and human health, including defense mechanisms against pathogens, antibiotic resistance, regulation pathways, genetic disorders and drug design. In our poster, we present the instrumentation and robotics available in the platform and a summary of results obtained during the last five years.

Keywords: High throughput, crystallization, pipeline 